\title{
Integral-field spectroscopy of (90482) Orcus-Vanth ${ }^{\star}$
}

\author{
B. Carry ${ }^{1,2,3,4}$, D. Hestroffer ${ }^{4}$, F. E. DeMeo ${ }^{2,5}$, A. Thirouin ${ }^{6}$, J. Berthier ${ }^{4}$, P. Lacerda ${ }^{7}$, B. Sicardy ${ }^{2,8,9}$, \\ A. Doressoundiram ${ }^{2}$, C. Dumas ${ }^{10}$, D. Farrelly ${ }^{11}$, and T. G. Müller ${ }^{12}$ \\ ${ }^{1}$ European Space Astronomy Centre, ESA, PO Box 78, 28691 Villanueva de la Cañada, Madrid, Spain \\ e-mail: benoit. carry@esa.int \\ 2 LESIA, Observatoire de Paris, CNRS, 5 place Jules Janssen, 92190 Meudon, France \\ 3 Université Paris 7 Denis-Diderot, 5 rue Thomas Mann, 75205 Paris Cedex, France \\ 4 IMCCE, Observatoire de Paris, UPMC, CNRS, 77 Av. Denfert Rochereau 75014 Paris, France \\ 5 Department of Earth, Atmospheric, and Planetary Sciences, MIT, 77 Massachusetts Avenue, Cambridge, MA 02139, USA \\ ${ }^{6}$ Instituto de Astrofísica de Andalucía, CSIC, Apt 3004, 18080 Granada, Spain \\ 7 Queen's University, Belfast, County Antrim BT7 1NN, Ireland \\ 8 Université Pierre et Marie Curie, 4 place Jussieu, 75252 Paris Cedex 5, France \\ 9 Institut Universitaire de France, 103 Bld Saint Michel, 75005 Paris, France \\ 10 Alonso de Córdova 3107, Vitacura, Casilla 19001, Santiago de Chile, Chile \\ 11 Utah State University, 0300 Old Main Hill, Logan, UT 84322, USA \\ 12 Max-Planck-Institut für extraterrestrische Physik (MPE), Giessenbachstrasse, 85748 Garching, Germany
}

Received 15 June 2011 / Accepted 27 August 2011

\begin{abstract}
Aims. We seek to constrain the surface composition of the trans-Neptunian object (90482) Orcus and its small satellite Vanth, as well as their mass and density.

Methods. We acquired near-infrared spectra (1.4-2.4 $\mu \mathrm{m}$ ) of (90482) Orcus and its companion Vanth using the adaptive-optics-fed integral-field spectrograph SINFONI mounted on Yepun/UT4 at the European Southern Observatory Very Large Telescope. We took advantage of a very favorable appulse (separation of only 4") between Orcus and the UCAC2 29643541 star $\left(m_{R}=11.6\right)$ to use the adaptive optics mode of SINFONI, allowing both components to be spatially resolved and Vanth colors to be extracted independently from Orcus.

Results. The spectrum of Orcus we obtain has the highest signal-to-noise ratio to date, and we confirm the presence of $\mathrm{H}_{2} \mathrm{O}$ ice in crystalline form, together with the presence of an absorption band at $2.2 \mu \mathrm{m}$. We set an upper limit of about $2 \%$ to the presence of methane, and 5\% for ethane. Since the methane alone cannot account for the $2.2 \mu \mathrm{m}$ band, the presence of ammonia is suggested to the level of a couple of percent. The colors of Vanth are found to be slightly redder than those of Orcus, but the large measurement uncertainties prevent us from drawing any firm conclusions about the origin of the pair (capture or co-formation). Finally, we reset the orbital phase of Vanth around Orcus, and confirm the orbital parameters derived by Brown and collaborators.
\end{abstract}

Key words. techniques: high angular resolution - techniques: imaging spectroscopy - Kuiper belt objects: individual: (90482) Orcus methods: observational

\section{Introduction}

Moons in the solar system are of high importance because they provide the most direct and precise way to derive the mass of the minor planets they orbit around (see Hilton 2002). Combined with volume estimates, their densities can be calculated, providing information about their composition and interior (e.g., Merline et al. 2002; Britt et al. 2002). They can subsequently help us to constrain the characteristics of the most pristine material of the solar system, and further our understanding of planetary system formation and dynamical evolution. In this valuable context, the trans-Neptunian binary (TNB) Orcus/Vanth system is of particular interest for the following reasons:

1. With an estimated albedo of $\sim 27 \%$ (Lim et al. 2010), Orcus is among the brightest known trans-Neptunian objects (TNOs), and has a diameter of about $850 \mathrm{~km}$.

* Based on observations collected at the European Southern Observatory Very Large Telescope (programs ID: 284.C-5044 and 384.C-0877).
2. Near-infrared spectroscopy of Orcus has revealed a surface rich in water ice in crystalline form (Fornasier et al. 2004; de Bergh et al. 2005; Trujillo et al. 2005; Barucci et al. 2008b; DeMeo et al. 2010). Moreover, Trujillo et al. (2005), Barucci et al. (2008b), and Delsanti et al. (2010) detected a weak band around $2.2 \mu \mathrm{m}$ that might be associated with either methane $\left(\mathrm{CH}_{4}\right)$ or ammonia $\left(\mathrm{NH}_{3}\right)$. The long-term stability of all ices are affected by high energy photon bombardment (causing photodissociation and sputtering), micrometeorite impacts, radioactive decay, and sublimation. Both methane and ammonia are expected to be destroyed by solar irradiation on short timescales (Strazzulla \& Palumbo 1998; Cooper et al. 2003; Cottin et al. 2003). Ammonia's presence, if confirmed, would thus require an active process to resupply the surface with ammonia, such as impact gardening or, more favorably, cryovolcanism (the ammonia lowers the melting temperature of water ice and hence favors such mechanism as highlighted by Cook et al. 2007). Bodies in the outer solar system that have methane on 
their surface have retained their atmospheres, which has important implications for its discovery on the surface of Orcus.

3. Recent radiometric measurements from ESA Herschel (as part of the key program "TNOs are Cool!"; see Müller et al. 2009) have been used to refine the size estimate of Orcus to $850 \pm 90 \mathrm{~km}$ (Lim et al. 2010). The diameter estimate will potentially be improved from the stellar occultations expected for upcoming years. Thus the improvement in the accuracy to which Vanth's orbit is known (based on the solution by Brown et al. 2010) will help us to determine the bulk density of Orcus.

We present here new spectro-imaging data obtained in 2010 that provide constraints on the composition of Orcus and the orbit of Vanth. We describe in Sect. 2 the observations, list in Sect. 3 the data reduction and spectral extraction steps, present in Sect. 4 the analysis of the colors and spectra of Orcus and Vanth, and detail in Sect. 5 the orbit computation and stellar occultation prediction.

\section{Observations}

The brightness contrast $\left(\Delta m_{V} \sim 2.6\right)$ and small apparent angular separation $\left(\sim 0.2^{\prime \prime}\right)$ between Orcus and its satellite Vanth require the use of a high angular-resolution camera/spectrograph to spatially resolve the system. This means observations have to be conducted in the visible from the Hubble Space Telescope (e.g., Brown et al. 2010), or in the near-infrared with ground-based telescopes equipped with adaptive optics $(\mathrm{AO})$ modules. The latter is of great interest for cold objects such as TNOs because many ices display strong absorption bands in the near-infrared (see Barucci et al. 2008a).

However, because adaptive-optics systems require a bright $\left(m_{V} \leq 15\right)$ reference source (a natural guide star: NGS) to correct the incident wavefront from the deformations induced by the atmospheric turbulence, study of TNOs from the ground with $\mathrm{AO}$ is generally limited to the brightest objects (e.g., Pluto or Haumea). The extension of these studies to fainter targets is possible thanks to two techniques. First, a laser beam can be projected into the atmosphere to create an artificial star of magnitude $m_{R} \sim 13.4$, called a laser guide star (LGS). However, because the laser beam is deflected on its way up by the atmospheric turbulence, the LGS position moves on the plane of the sky in a random pattern (corresponding to low orders of the turbulence, called tip-tilt). Hence, a natural close-by star must be monitored to correct the wavefront for the motion of the LGS. Because the requirement on these reference stars (called tip-tilt star: TTS) are less strict (angular distance and brightness) than for NGS, several TNOs have already been observed this way (e.g., Brown et al. 2006; Dumas et al. 2011).

The second technique consists of computing close encounters (separations smaller than about $30^{\prime \prime}$ ) on the plane of the sky between the object of interest and a star suitable as a NGS (e.g., Berthier \& Marchis 2001). These events are called appulses. On 2010 February 23 UT, Orcus had a particularly favorable appulse with the star UCAC2 $29643541\left(m_{R}=11.6\right)$ at an angular separation of only $4^{\prime \prime}$. We thus observed it in Service Mode (program ID: 284.C-5044) at the European Southern Observatory (ESO) Very Large Telescope (VLT) with the near-infrared integralfield spectrograph SINFONI (Eisenhauer et al. 2003; Bonnet et al. 2004). Observations were realized simultaneously in the atmospheric $H$ and $K$ bands $(1.45-2.45 \mu \mathrm{m})$ using the $H+K$ grating of SINFONI, providing a spectral resolving power $R$ of about 1500 . We used a plate scale of $50 \times 100$ mas/pixel,
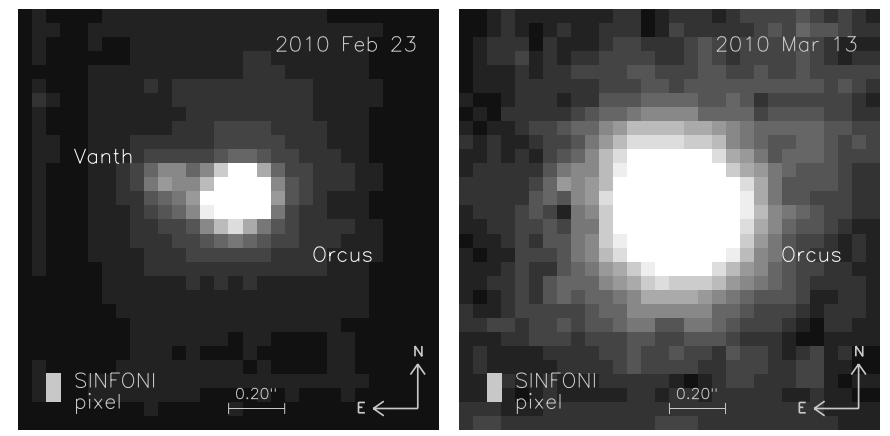

Fig. 1. Two images of the Orcus-Vanth system, obtained by summing all individual observations and stacking the resulting cube for the whole range of wavelengths. Left: our 2010 February 23 UT observations using the appulse NGS AO correction, representing a total integration time of $4050 \mathrm{~s}$. Both Orcus and Vanth are easily separable in the image. Right: our 2010 March 13 UT observations using the LGS AO correction, without TTS reference (see text), corresponding to a total integration time of $5400 \mathrm{~s}$. The angular resolution provided in that mode forbids the detection of Vanth, whose flux, spread over many pixels, is hidden within the background noise.

associated with a $3^{\prime \prime} \times 3^{\prime \prime}$ field of view. We alternated observations of Orcus and the nearby sky in a jitter pattern to allow optimal sky subtraction, being cautious to avoid the NGS (4").

Unfortunately, the AO module of SINFONI had not been designed to offer differential tracking (i.e., NGS fixed on the plane of the sky, field of view following a target with non-sidereal motion). We thus had to set the duration of integrations as a compromise between the slew of Orcus on the detector plane and the count level reached on Vanth $\left(m_{V} \sim 21.6\right)$. We used individual exposures of $150 \mathrm{~s}$ to theoretically ${ }^{1}$ achieve an average signalto-noise ratio $(S / N)$ of 1 on Vanth over $H$ band. In return, during a single exposure, Orcus moved by $-0.109^{\prime \prime}$ in right ascension and $0.039^{\prime \prime}$ in declination, distorting its apparent shape, which was thus elongated along the SE-NW direction as is clearly visible in Fig. 1.

Atmospheric conditions at the time of the observations were very good, with an average seeing of $0.8^{\prime \prime}$ and a coherence time ranging from 7 to $20 \mathrm{~ms}$. Orcus was close to zenith during the observations with an airmass ranging from 1.05 to 1.4. This allowed the AO system to provide an optimal correction, resulting in a spatial resolution close to the diffraction limit of the telescope (the full width at half maximum $(F W H M)$ of Orcus was $85 \times 100$ mas in $K$-band).

We also report here on some test observations of Orcus performed on 2010 March 13 UT at the ESO VLT (prog. ID: 384.C-0877) in the so-called "seeing enhancer" mode. This mode consists of closing the AO loop on a LGS, but without providing any TTS, Orcus itself being too faint $\left(m_{V} \sim\right.$ 19.7) to be used as a TTS (as opposed to targets such as Haumea, see Dumas et al. 2011, for instance). Hence, only the higher orders of the atmospheric turbulence are corrected (i.e., there is no tip-tilt correction). The advantage of this mode is that we can perform differential tracking and therefore take longer exposures (600 s). The instrument settings and observing strategy were otherwise similar to those for February observations.

Atmospheric conditions were worse during March observations, with an average seeing of $0.9^{\prime \prime}$, and coherence time of about $3 \mathrm{~ms}$. However, the quality of the correction provided by the $\mathrm{AO}$ in that mode is intrinsically lower than for the

${ }^{1}$ Computation made using ESO Exposure Time Calculator. 
appulse: the $F W H M$ of Orcus was $0.38^{\prime \prime}$ (still representing an improvement by a factor of $\approx 2$ with respect to seeing-limited observations). Despite the shape of Orcus being elongated by its apparent displacement in February, the quality of the data was superior (with a shorter exposure time) to the March data, where the spread of its light is directly related to the lower AO correction achieved. This highlights the advantage of searching for favorable appulses for faint moving targets to use bright NGS as reference for the adaptive-optics correction.

\section{Data reduction and analysis}

We used the SINFONI pipeline (Modigliani et al. 2007) version 2.0.5 to perform the basic data reduction: bad pixel removal, flat fielding correction, subtraction of the sky background from the jittered observations, and wavelength calibration with Xenon-Argon-Krypton lamps (see Guilbert et al. 2009, for a complete description of the procedure on other faint TNOs). Default parameters were used, except for in the "jitter" recipe where we set the parameters scales.sky to true and density to three to achieve optimal sky-background correction. This provided us with 27 and 9 individual cubes (two spatial plus one spectral dimensions) of Orcus/Vanth for February and March observations, respectively.

We then computed the average centroid position of Orcus for each individual observation by stacking the cubes along wavelength. We used this information to shift and add all the cubes into a single one for each date, corresponding to equivalent exposure times of $4050 \mathrm{~s}$ and $5400 \mathrm{~s}$. We then re-aligned all the wavelength slices of the cube because the centroid position of Orcus was not constant with wavelength but rather experienced a slow drift caused by the differential atmospheric refraction as described in Carry et al. (2010).

We then extracted the respective spectra of Orcus and Vanth by adjusting (using MPFIT least square algorithm of Markwardt 2009), for each wavelength, a model $\mathcal{I}$ composed of a linear background and two Moffat functions describing both components

$\mathcal{I}(x, y)=\mathcal{F}_{\mathrm{o}}(x, y)+\mathcal{F}_{\mathrm{v}}(x, y)+a x+b y+c$,

where $\mathcal{F}_{i}$ are the two Moffat functions, representing Orcus $\left(\mathcal{F}_{\mathrm{o}}\right)$ and Vanth $\left(\mathcal{F}_{\mathrm{v}}\right)$, defined by

$\mathcal{F}_{i}(x, y)=f_{i} \cdot\left[\begin{array}{c}1+\left(\left(x-x_{i}^{c}\right) \frac{\cos \theta}{\sigma_{x}}-\left(y-y_{i}^{c}\right) \frac{\sin \theta}{\sigma_{y}}\right)^{2} \\ +\left(\left(x-x_{i}^{c}\right) \frac{\sin \theta}{\sigma_{x}}+\left(y-y_{i}^{c}\right) \frac{\cos \theta}{\sigma_{y}}\right)^{2}\end{array}\right]^{-\alpha}$,

where $x, y$ are the frame spatial dimensions, $f_{i}$ is the peak level of each Moffat function centered on the coordinates $\left(x_{i}^{c}, y_{i}^{c}\right) . \sigma_{x}$, $\sigma_{y}$ are the half-width at half maximum (HWHM) along two perpendicular directions, making an angle $\theta$ with the detector $x$ direction, and $\alpha$ is the power-law index of the Moffat functions. The final spectrum was cleaned for bad points using a $3 \sigma$ median smoothing procedure.

The advantage of this method is to provide the spectra of both components as well as their relative astrometry. We discuss both points in the subsequent sections.

\section{Spectral analysis}

\subsection{The surface composition of Orcus}

Figure 2 compares our new spectrum of Orcus to that of Barucci et al. (2008b). The overall spectral shape reveals the presence

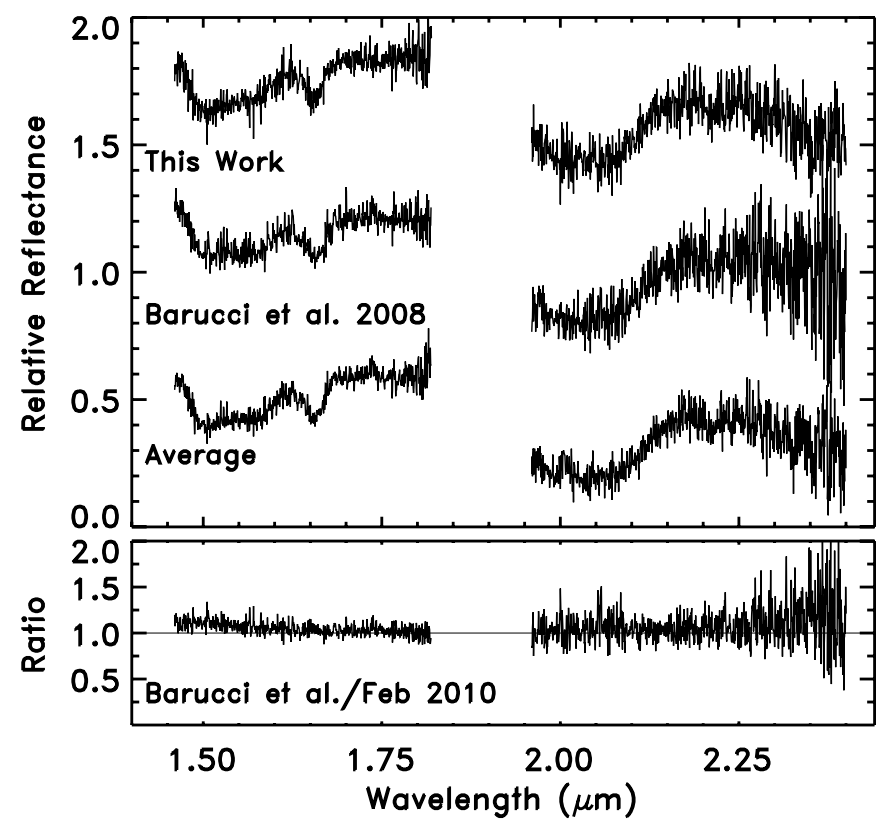

Fig. 2. Upper panel: spectra of Orcus in the $H$ and $K$ bands from this work (top) and Barucci et al. (2008b) (middle). The bottom spectrum is an average of the two. The spectra are normalized to 1.0 at $1.75 \mu \mathrm{m}$ and are shifted by $+0.65,0$, and -0.4 . The spectrum taken in March 2010 was significantly noisier than the two shown here so we neither plot it nor use it in our analysis. Lower panel: the ratio of the Barucci et al. data to data from this work, which shows little difference between the spectra apart from a small flux difference shortward of $1.65 \mu \mathrm{m}$.

of water ice, dominated by the crystalline form as already addressed in previous work (Fornasier et al. 2004; de Bergh et al. 2005; Trujillo et al. 2005; Barucci et al. 2008b; Guilbert et al. 2009; Delsanti et al. 2010; DeMeo et al. 2010).

The ratio of these two spectra, shown in the bottom part of Fig. 2, reveals their similarity, although we note a difference in the overall flux level $(\sim 10 \%)$ shortward of $\sim 1.65 \mu \mathrm{m}$. This difference does not appear to be related to any variation in $\mathrm{H}_{2} \mathrm{O}$ (amount or grain size) because it is present shortward of $1.5 \mu \mathrm{m}$. Potential explanations include instrumental effects or differences of the standard stars.

We confirm the detection of a feature near $2.2 \mu \mathrm{m}$ with a band center located at $2.209 \pm 0.002 \mu \mathrm{m}$ and a band depth of $9 \pm 2 \%$ that previous works have attributed to $\mathrm{CH}_{4}, \mathrm{NH}_{3}$, or $\mathrm{NH}_{4}^{+}$. We combined the Barucci et al. spectrum with ours to slightly increase the overall $S / N$. We did not include the spectra of both Delsanti et al. (2010) or DeMeo et al. (2010) because the quality of these data were significantly lower. This average spectrum is used for all of the analysis reported in this section.

Current volatile retention models (e.g., Schaller \& Brown 2007; Levi \& Podolak 2009) predict that $\mathrm{CH}_{4}$ is unstable on Orcus' surface over its lifetime, although Orcus' intermediate size among TNOs place it closer to the retention boundary than most other objects and provide us with an opportunity to test these models and perhaps place some constraints on the assumptions therein. An important step in understanding Orcus' surface composition is thus a search for weak bands hidden in the spectrum near the detection limit. While many species could potentially exist in small quantities on the surface, the lack of multiple strong bands makes their identification difficult. Here we focus on searching for methane bands in the spectrum primarily because of the abundance of strong bands in the appropriate wavelength regime, but also because of the important implications 


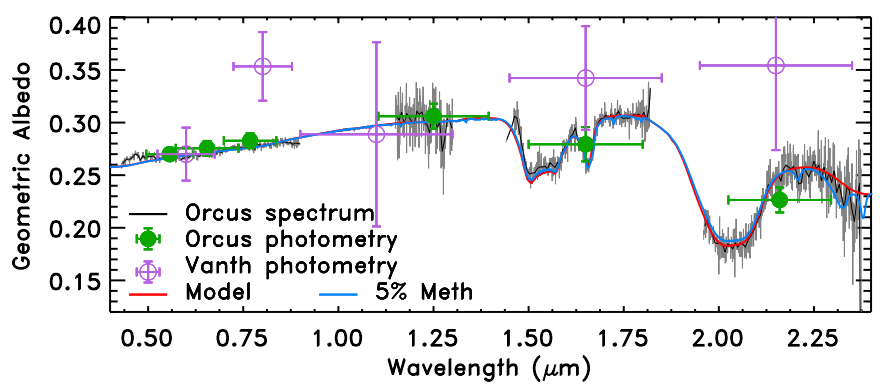

Fig. 3. Plotted here is the average spectrum of Orcus from our work and Barucci et al. together with the visible data from DeMeo et al. (2009), scaled to the visible albedo estimated by Lim et al. (2010). Overplotted are the basic model in red (\#1 in Table 1) and model with additional $5 \%$ methane in blue (\#2,ibid). Also shown in purple are the visible and near-infrared colors of Vanth. The $V, I$, and $J$ band measurements are from Brown et al. (2010). The colors of Vanth are normalized to Orcus' spectrum at 0.6 microns.

a detection would have on our understanding of the surface conditions of these bodies and the criteria for volatile retention.

First, to remove the dominant signature of the crystalline water ice from the spectrum we model the composition of Orcus using a code based on radiative transfer theory (Hapke 1993) using optical constants of laboratory materials for inputs. We use optical constants of $\mathrm{H}_{2} \mathrm{O}$ ice in both crystalline and amorphous form (at $40 \mathrm{~K}$ and $38 \mathrm{~K}$ from Grundy \& Schmitt 1998; and Schmitt et al. 1998, respectively) and Titan and Triton tholin (Khare et al. 1984, 1993). The temperature of the $\mathrm{H}_{2} \mathrm{O}$ optical constants are appropriate because the blackbody temperature at $39 \mathrm{AU}$ is about $43 \mathrm{~K}$ and Pluto's surface temperature (with a similar semi-major axis) is measured to be $40 \pm 2 \mathrm{~K}$ (Tryka et al. 1994). Triton and Titan tholins are used as representative material that aid in fitting the spectrum, because optical constants are available for these materials. However, they could be replaced with different materials, such as other organics that have similar spectral properties. The models we present here (see Table 1 and Fig. 3) are based on the recent analysis by DeMeo et al. (2010) but differ slightly because they are based on the recent reevaluation of the albedo of Orcus from $0.20 \pm 0.03$ (Stansberry et al. 2008 ) to $0.27 \pm 0.06$ by Lim et al. (2010). We reduced the fraction of amorphous water ice to about $10 \%$, increasing the crystalline $\mathrm{H}_{2} \mathrm{O}$ by the same amount to provide a closer fit of the $1.65 \mu \mathrm{m}$ band and adjusted the cosine asymmetry factor (Hapke 1993 ) to properly fit the data's higher albedo. The spectrum was then divided by the model \#1, without methane (Table 1). We created a program in IDL designed to fit Gaussians to potential features in designated wavelength regions. The program was set to search in the regions near $1.67,1.72$, and $2.2 \mu \mathrm{m}$, where methane absorbs strongly (Quirico \& Schmitt 1997). We did not search for the band near $1.80 \mu \mathrm{m}$ because of poor telluric correction in this wavelength range, nor the bands near $2.32 \mu \mathrm{m}$ and $2.43 \mu \mathrm{m}$ owing to a decreasing $S / N$ at wavelengths longer than $\approx 2.3 \mu \mathrm{m}$ from low detector sensitivity. A least squares minimization (Markwardt 2009) was used to find the best-fit center, width, and depth of the bands.

The results of the Gaussian fits are listed in Table 2, and plots of the fits are shown in Fig. 4. We find a Gaussian fit near the $1.67 \mu \mathrm{m}$ feature, although the center is at $1.654 \mu \mathrm{m}$ indicating that it is residual crystalline $\mathrm{H}_{2} \mathrm{O}$ that was not removed by the division of the data by the model. We do not find a band at $1.67 \mu \mathrm{m}$ nor at $1.72 \mu \mathrm{m}$. The depth of the $2.209 \mu \mathrm{m}$ Gaussian fit is $9.5 \pm 2.3 \%$. While we do not fit a Gaussian to the $2.32 \mu \mathrm{m}$
Table 1. Material present in the models of surface composition.

\begin{tabular}{cccc}
\hline \hline & Material & Amount $(\%)$ & Grain size $(\mu \mathrm{m})$ \\
\hline Model 1 & Crystalline $\mathrm{H}_{2} \mathrm{O}$ & 60 & 18 \\
& Amorphous $\mathrm{H}_{2} \mathrm{O}$ & 10.5 & 18 \\
& Titan tholins & 2 & 10 \\
& Triton tholins & 6 & 10 \\
& Blue compound & 22.5 & 10 \\
Model 2 & Crystalline $\mathrm{H}_{2} \mathrm{O}$ & 60 & 18 \\
& Amorphous $\mathrm{H}_{2} \mathrm{O}$ & 5.5 & 18 \\
& Titan tholins & 2 & 10 \\
& Triton tholins & 6 & 10 \\
& Blue compound & 22.5 & 10 \\
& Methane & 5 & 100 \\
\hline
\end{tabular}

Table 2. Parameters for weak bands.

\begin{tabular}{lcccc}
\hline \hline Species & $\begin{array}{c}\lambda_{\mathrm{e}} \\
(\mu \mathrm{m})\end{array}$ & $\begin{array}{c}\lambda_{\mathrm{m}} \\
(\mu \mathrm{m})\end{array}$ & $\begin{array}{c}\Delta \lambda \\
(\mathrm{nm})\end{array}$ & $\begin{array}{c}\text { Depth } \\
(\%)\end{array}$ \\
\hline $\mathrm{CH}_{4}$ & 1.670 & $1.654 \pm 0.004$ & $8 \pm 5$ & $4.1 \pm 1.9$ \\
$\mathrm{CH}_{4}$ & 1.724 & - & - & $<2$ \\
$\mathrm{CH}_{4}$ & 2.208 & $2.209 \pm 0.002$ & $6 \pm 2$ & $9.5 \pm 2.3$ \\
$\mathrm{C}_{2} \mathrm{H}_{6}$ & 2.274 & - & - & $<2$ \\
$\mathrm{C}_{2} \mathrm{H}_{6}$ & 2.314 & - & - & $<2$ \\
\hline
\end{tabular}

Notes. Band centers $\left(\lambda_{\mathrm{e}}\right)$ of the strongest $\mathrm{CH}_{4}$ bands from Quirico \& Schmitt (1997). We list the band center $\left(\lambda_{\mathrm{m}}\right)$, width $(\Delta \lambda)$, and depth, from the Gaussian fit to our data at these wavelengths if detected.

feature, we do note that the reflectance of the data is lower in this region with respect to the model that excludes $\mathrm{CH}_{4}$ (\#1) suggesting there is a compound absorbing in this region, though not necessarily $\mathrm{CH}_{4}$.

The question that remains is "can the band at $2.2 \mu \mathrm{m}$ be produced by methane even though we do not detect any other bands?" The relative band depths of the 1.67 and $1.72 \mu \mathrm{m}$ features compared to the $2.2 \mu \mathrm{m}$ feature are $95 \%$ and $88 \%$, respectively, assuming a grain size of $200 \mu \mathrm{m}$ (the relative depths do not change significantly with variations in grain size of $\pm 100 \mu \mathrm{m})$. We should clearly be able to detect all three bands, if the $2.2 \mu \mathrm{m}$ feature were due to methane only (as visible in Fig. 3 by comparing the spectrum of Orcus with model \#2, including $5 \%$ of methane to account for the $2.2 \mu \mathrm{m}$ feature), thus we place a limit of a maximum fraction of about $2 \%$ for methane at the surface. Our constraint of $2 \%$ is lower than the constraint set in DeMeo et al. (2010) partly because of the higher quality of our data, but also because the bands predicted by the $5 \%$ methane model at the higher albedo are weaker than for the lower albedo model. The majority (or all) of the $2.2 \mu \mathrm{m}$ absorption must therefore be due to either hydrated ammonia, ammonium, as suggested in previous works, or another yet unknown compound that absorbs in this region. Since $\mathrm{H}_{2} \mathrm{O}: \mathrm{NH}_{3}$ and $\mathrm{NH}_{4}^{+}$do not have any other distinguishing features in the wavelength range of our data we can neither confirm nor exclude their presence.

We also search for bands at 2.274 and $2.314 \mu \mathrm{m}$, the positions of the strongest bands of ethane (Quirico \& Schmitt 1997). Ethane is a by-product of methane and its potential presence on Orcus was suggested by models fitted to data by Delsanti et al. (2010). We do not find any bands at the locations of ethane absorption within the limits of the quality of our data (which is the best to date). Either there is no ethane (less than about $5 \%$, 


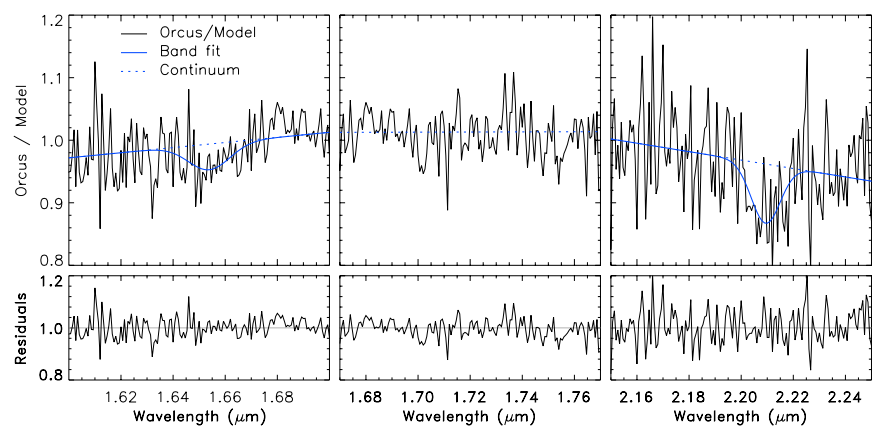

Fig. 4. Gaussian fits to the data to search for weak bands. Two bands of approximately $4 \%$ and $9 \%$ are detected around 1.65 and $2.2 \mu \mathrm{m}$, respectively (see Table 2). No band with any depth weaker than about $2 \%$ is detectable around $1.72 \mu \mathrm{m}$.

Table 3. Relative magnitude difference of Orcus and Vanth.

\begin{tabular}{lcccc}
\hline \hline Filter & Orcus $^{1}$ & $\Delta \mathrm{mag}$ & Flux ratio & Refs. \\
\hline$V$ & $19.36 \pm 0.05$ & $2.61 \pm 0.10$ & $9.0 \pm 0.9$ & 1 \\
$I$ & $18.63 \pm 0.05$ & $2.31 \pm 0.10$ & $8.6 \pm 2.9$ & 1 \\
$J$ & $20.64 \pm 0.04$ & $2.66 \pm 0.34$ & $11.5 \pm 0.5$ & 1 \\
$H$ & $21.65 \pm 0.03$ & $2.34 \pm 0.12$ & $11.6 \pm 1.3$ & 2 \\
$K \mathrm{~s}$ & - & $2.07 \pm 0.24$ & $14.9 \pm 3.2$ & 2 \\
\hline
\end{tabular}

References. (1) Brown et al. (2010); (2) this work.

depending on grain size, within the limits of detection with our data) on Orcus' surface or, less likely, there is a concentration of ethane on the part of the surface observed by Delsanti et al. that we did not observe. We also check for the possible presence of other volatile compounds: $\mathrm{CO}_{2}$ (three narrow features near $2 \mu \mathrm{m}), \mathrm{CO}(1.578 \mu \mathrm{m}$, we do not search at $2.352 \mu \mathrm{m}$ because of the poor quality of the data), $\mathrm{N}_{2}(2.15 \mu \mathrm{m})$, and methanol $(2.27 \mu \mathrm{m})$. We do not detect any of these features in our data.

\subsection{The colors of Vanth}

The spectrum of Vanth that we extracted had a very low $S / N$, hence we drastically reduced its spectral resolution and we only report in Table 3 its magnitude differences from Orcus in $H$ - and $K$-band. Figure 3 compares the visible and near-infrared colors of Vanth to those of Orcus, scaled to the visible albedo of Orcus from Lim et al. (2010). Although the errors are large, the colors indicate that Vanth is slightly redder in both the visible wavelength regime as reported by Brown et al. (2010) and the nearinfrared as indicated by our data. While the colors of Vanth do not suggest that there is a strong presence of water ice similar to that seen on Orcus, the possibility cannot be ruled out within the uncertainty of the data. As already addressed by Brown et al., it is impossible to draw any conclusions about the origin of this color difference to distinguish between a capture or collision formation scenario. Even if Vanth was indeed found to have colors inconsistent with water ice, this might may not exclude a collision formation scenario.

\section{Orbital characterization}

The relative orbit of the Orcus/Vanth system was previously determined by Brown et al. (2010) from observations conducted using the Hubble Space Telescope (HST), mainly with the high resolution channel of the Advanced Camera for Surveys (ACS), over the period 2005-2007. Three years after, the uncertainty in

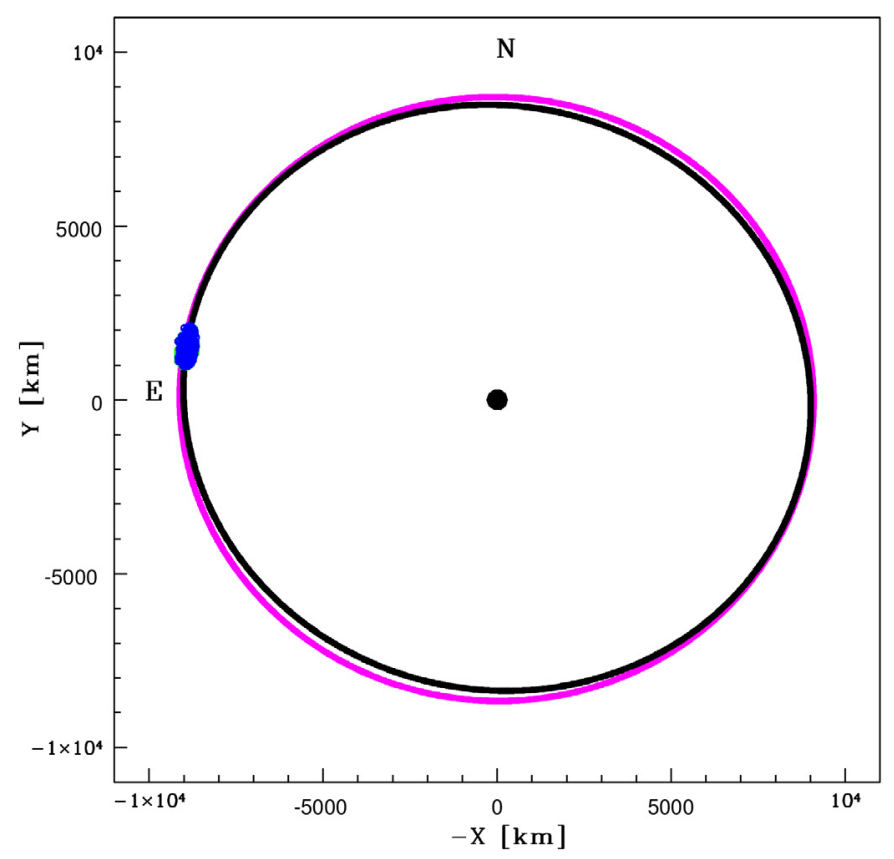

Fig. 5. Predicted position of Vanth relative to Orcus on 2010 Feb. 23.237, from the extrapolation of the initial 2004-2007 data by Brown et al. (2010). The two curves reprensent two possible solutions with symmetric pole solutions. The dots in the Eastern part of the frame correspond to the predicted positions at the time of the SINFONI observation for all retained orbital solutions given in Fig. 6.

the orbital period leads to a position uncertainty for Vanth along its orbit of about $15^{\circ}$, corresponding to $2000 \mathrm{~km}$ (Fig. 5). With one additional point for the relative position of the components after three years, corresponding to some 2000 revolutions of the pair, it is possible to refine the orbit, especially the orbital period, which in turn allows us to refine the position prediction for future observations, in particular stellar occultations.

In this section we present the relative astrometry measurements, the subsequent orbit computation, and the consequences for the predictions of stellar occultations.

\subsection{Astrometry measurements}

To extract the relative astrometry of Orcus and Vanth, we first stacked the spectrocubes along the wavelength dimension to obtain two frames of higher SNR, one at each epoch. We then used the image model $\mathcal{I}$ presented in Sect. 3, the astrometry being defined by the relative position of the Moffat function centers.

As visible in Fig. 1, Vanth is easily separable from Orcus on the image obtained in February, and we estimate the accuracy of its position to be about 25 mas, corresponding to a fourth of a pixel on the SINFONI detector. However, our observing date, which was imposed by the stellar appulse, was not optimal for taking full advantage of the parallactic effect and removing the ambiguity in the relative orbit inclination (see the two curves in Fig. 5). On the other hand, the moderate spatial resolution (FWHM of 380 mas) achieved in March did not allow the localization of Vanth, whose flux was spread over a large area and diluted by the background noise (Fig. 1).

We also use the astrometric measurements given in Brown et al. (2010), although, after analyzing the public HST data, it is clear that the dates given in Brown et al. (2010) are wrong by a constant offset of half a day (as confirmed by D. Ragozzine 
Table 4. Astrometric data for the relative position of Orcus/Vanth used to reconstruct their mutual orbit.

\begin{tabular}{cccccccc}
\hline \hline $\begin{array}{c}\text { Date } \\
(\mathrm{JD})\end{array}$ & $\begin{array}{c}\Delta X^{\ddagger} \\
\left({ }^{\prime \prime}\right)\end{array}$ & $\begin{array}{c}\Delta Y^{\ddagger} \\
\left({ }^{\prime}\right)\end{array}$ & $\begin{array}{c}\Delta \\
(\mathrm{AU})\end{array}$ & $\begin{array}{c}r \\
(\mathrm{AU})\end{array}$ & $\begin{array}{c}\alpha \\
\left({ }^{\circ}\right)\end{array}$ & $\begin{array}{c}\delta \\
\left({ }^{\circ}\right)\end{array}$ & Instr. \\
\hline 2453687.66400 & 0.206 & -0.147 & 47.811 & 47.702 & 144.039 & -4.356 & ACS \\
2454040.36900 & 0.226 & -0.111 & 48.075 & 47.752 & 144.813 & -4.729 & ACS \\
2454044.36600 & -0.258 & -0.005 & 48.013 & 47.752 & 144.839 & -4.766 & ACS \\
2454051.57900 & -0.006 & -0.243 & 47.898 & 47.753 & 144.871 & -4.828 & ACS \\
2454056.08900 & -0.036 & 0.240 & 47.824 & 47.754 & 144.882 & -4.865 & ACS \\
2454066.14600 & 0.053 & 0.240 & 47.659 & 47.755 & 144.882 & -4.938 & ACS \\
2454080.33600 & -0.030 & -0.244 & 47.433 & 47.757 & 144.824 & -5.019 & ACS \\
2454416.29300 & -0.263 & -0.024 & 47.968 & 47.801 & 145.703 & -5.304 & NICMOS3 \\
2454439.78000 & 0.245 & 0.078 & 47.585 & 47.804 & 145.696 & -5.473 & WFPC2 \\
\hline 2455250.73686 & 0.260 & 0.052 & 46.954 & 47.895 & 146.287 & -6.396 & SINFONI \\
\hline
\end{tabular}

Notes. ${ }^{(\dagger)}$ The first block is reproduced from Brown et al. (2010) with the corrected values for the dates (see text). The second bloc corresponds to our appulse observation on 2010 February 23. ${ }^{(\ddagger)}$ Positions are positive through east and north.

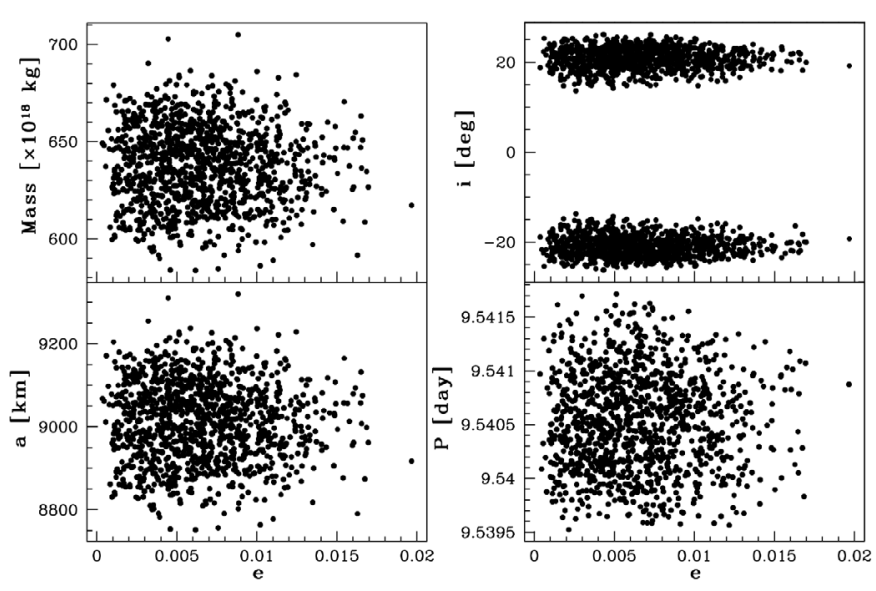

Fig. 6. Elliptical orbital elements: system mass $(\mathcal{M})$, semi-major axis $(a)$, inclination $(i)$, and period $(P)$, plotted as a function of the eccentricity $(e)$. Each point represents a different orbital solution that fits the astrometry measurements in Table 4 . See Table 5 for a summary of the orbital elements.

and M. Brown, personal communication). All the astrometric measurements are reproduced in Table 4, along with the observation circumstances: heliocentric distance $(\Delta)$ and range to observer $(r)$, right ascencion $(\alpha)$, and declination $(\delta)$.

\subsection{Orbit improvement}

With our additional astrometric data, we can improve the orbital parameters, reset the orbital phase of Vanth, and, taking advantage of the time leverage, provide tighter constrains than possible before of the orbital period. We computed an improved Keplerian orbit using a statistical inversion algorithm (Hestroffer et al. 2005), which allows us to probe a large portion of the orbital parameter space. We show in Fig. 6 all the solutions that are consistent with the observations, within the measurement uncertainties, and list the resulting improved orbital and physical parameters in Table 5 .

As mentioned before, the position of Vanth in its orbit on 2010 Feb. 23 (see Fig. 5) did not allow us to remove the ambiguity of the inclination of the orbit $\left( \pm 21^{\circ}\right.$ with respect to the average plane-of-the-sky). However, the orbital pole solution that is almost perpendicular to the ecliptic $\left(P_{\mathrm{A}}\right.$, corresponding
Table 5. Orbital elements and physical parameters for the Orcus/Vanth system.

\begin{tabular}{lcccc}
\hline \hline Qty. & Value & Uncert. & Description & \\
\hline$a$ & 9030 & 89 & $\mathrm{~km}$ & Semi-major axis \\
$e$ & 0.007 & 0.003 & - & Eccentricity $^{1}$ \\
$i$ & $( \pm) 21$ & 2 & deg. & Inclination $^{1}$ \\
$\Omega$ & 144 & 63 & deg. & Ascending node ${ }^{1}$ \\
$n$ & 37.732 & 0.002 & deg/day & Mean motion \\
\hline$P$ & 9.5406 & 0.0004 & day & Orbital period \\
$P_{\mathrm{A}}$ & $(321,-2)$ & 3 & deg. & Pole A coordinates \\
$P_{\mathrm{B}}$ & $(340,+38)$ & 3 & deg. & Pole B coordinates $^{2}$ \\
\hline $\mathcal{M}$ & 641 & 19 & $10^{18} \mathrm{~kg}$ & Total mass $^{3}$ \\
$\mathcal{D}$ & 850 & 90 & $\mathrm{~km}$ & Diameter $^{3}$ \\
$D_{\mathrm{o}}$ & 807 or 761 & 100 & $\mathrm{~km}$ & Orcus diameter $^{4}$ \\
$D_{\mathrm{v}}$ & 267 or 378 & 100 & $\mathrm{~km}$ & Vanth diameter $^{4}$ \\
$\rho$ & 2250 or 2470 & 885 & $\mathrm{~kg} \mathrm{~m}^{-3}$ & Bulk density $^{2}$ \\
\hline
\end{tabular}

Notes. Uncertainties are the formal $1 \sigma$ confidence intervals (from Fig. 6) and do not account for possible systematics (that arise from model incompleteness, e.g., neglecting Orcus $J_{2}$ which is unknown).

(1) In the tangent plane. ${ }^{(2)}$ Pole coordinates are given in the ecliptic J2000 reference frame. ${ }^{(3)}$ Volume-equivalent from radiometry (see Lim et al. 2010). ${ }^{(4)}$ Assuming alternatively $p_{\mathrm{v}}=p_{\mathrm{o}}$, or $p_{\mathrm{o}}=0.27$ and $p_{\mathrm{v}}=0.12$.

to $\left.i=+21^{\circ}\right)$, coordinates ECJ2000 $\left(321^{\circ},-2^{\circ}\right)$, is statistically more probable given the observable data (see Fig. 6), although the other solution $\left(P_{\mathrm{B}}\right)$ inclined at about $52^{\circ}$ from the ecliptic, coordinates ECJ $2000\left(340^{\circ},+38^{\circ}\right)$, provides very similar residuals and cannot yet be ruled out.

All the possible solutions that we find have low eccentricities $(e \leq 0.02)$. Brown et al. (2010) previously highlighted the nearly circular nature of Vanth's orbit around Orcus, although we find here several solutions with higher eccentricities than the upper limit they reported $(0.0036,1 \sigma$ deviation to the data). Similarly, we determine an average orbital period that is longer than the value of Brown et al. (2010) (consistent with Ortiz et al. 2011, however), their shorter period being still possible, although less likely. These differences might originate from the circular assumption about the orbit of Vanth used by Brown et al. In any case, since the eccentricity of the orbit is small, it suggests that the tides have circularized the orbit, and subsequently that the satellite is also in synchronous rotation. The time for the 
circularisation of the orbit of a satellite in such a system can be short, on the order of $\tau_{\mathrm{c}} \sim\left(a / R_{2}\right)^{5} n^{-1} \sim 10^{6}$ years (Ferraz-Mello et al. 2008). Another reasonable consequence of such a tidal evolution is that the orbital plane is aligned to the equator of the primary. In this case, we would currently see Orcus under a small aspect angle (roughly $10^{\circ}$ and $30^{\circ}$ for $P_{\mathrm{A}}$ and $P_{\mathrm{B}}$, respectively).

The circular orbit might also be caused, independently of tidal effects, by a Kozai resonance $\left(\sqrt{1-e^{2}} \cos i=\right.$ const., Kozai 1962), in which case the inclination of Vanth's orbit with respect to Orcus' equator can be large. This also means that the direction of the spin for the primary is unconstrained and that its aspect angle can be much larger. We favor here the first solution $\left(P_{\mathrm{A}}\right)$ as it is slightly more probable statistically, and is consistent with the low amplitude of the variations present in the optical lightcurve of Orcus (0.04 mag, see Thirouin et al. 2010, and references therein).

The total mass $\mathcal{M}$ is derived to high precision from Kepler's third law, considering that this dynamical system is quite isolated over the observational timescale, supported by the two-body fit. The recent size determined using ESA Herschel data by Lim et al. (2010) allows us to evaluate the volume, hence density, of the components. Because the components are not resolved by the thermal infrared pixels, one can only derive a radiometric volume-equivalent diameter $\mathcal{D}$ for the system, and subsequently model-dependent values for the density. This effective diameter can be simply related to the volume-equivalent diameter of each component by considering their projected apparent surface $\mathcal{D}^{2}=$ $D_{\mathrm{o}}^{2}+D_{\mathrm{v}}^{2}$. If we assume that the two components are of similar density, one eventually gets a rough estimate of the bulk density

$\rho=\frac{6 \mathcal{M}}{\pi \mathcal{D}^{3}} \frac{\left(1+\mathcal{R}^{2}\right)^{3 / 2}}{\left(1+\mathcal{R}^{3}\right)}$,

where the size ratio of the bodies $\mathcal{R} \equiv D_{\mathrm{v}} / D_{\mathrm{o}}=\sqrt{\frac{p_{\mathrm{o}}}{p_{\mathrm{v}}}} 10^{-0.2 \Delta m}$ essentially depends on their apparent magnitude difference $(\Delta m \approx 2.4 \pm 0.4$, see Table 3$)$, modulated by their unknown albedo ratio. Following Brown et al. (2010), we consider two possible albedos for Vanth: firstly 0.27 (i.e., equal to that of Orcus, corresponding to co-formation mechanisms, as described in Sect. 5.3), and secondly 0.12, typical of TNOs exempt of water ice (for capture mechanisms, as in Sect. 5.3). We find mass ratios of 30 and 8 , for the equal-albedo and differentalbedo assumptions respectively, close to the estimates of Brown et al. (2010) and Ortiz et al. (2011). On the basis of the refined volume-equivalent diameter of the Orcus/Vanth system by Lim et al. (2010), which is $10 \%$ smaller than the estimate of Brown et al. (2010), we find a density of about $2.3 \pm 0.8 \mathrm{~g} / \mathrm{cc}$. In any case, the uncertainty in the bulk density is dominated by the uncertainty in the size determination, which cannot be more accurate than about $10 \%$ (Lim et al. 2010). The relative precision in the bulk density cannot therefore be smaller than about $30 \%$. More accurate knowledge of the spin properties of Orcus, and of its shape are now required to improve its size, hence volume, estimate.

\subsection{Formation mechanism}

Trans-Neptunian binaries can be used to impose constraints on various models of solar system formation evolution, e.g., to constrain Neptune's migration history (Murray-Clay \& Schlichting 2011). Several formation mechanisms have been proposed and each leads to different predictions for the physical and orbital properties of TNBs. These formation mechanisms can, broadly, be broken down into four classes: collision (Weidenschilling 2002), rotational fission (Ortiz et al. 2011), capture (e.g., Goldreich et al. 2002; Funato et al. 2004; Astakhov et al. 2005; Lee et al. 2007; Gamboa Suárez et al. 2010), and gravitational collapse (Nesvorný et al. 2010).

In the collision model of Weidenschilling (2002), two objects collide inside the Hill sphere of a third object. These objects then fuse into a single object thereby producing a binary. The capture models of Goldreich et al. (2002) rely on two objects interpenetrating their mutual Hill sphere and then being stabilized either through dynamical friction (the $\mathrm{L}^{2}$ mechanism) or through a scattering event with a third, similarly sized, object (the $\mathrm{L}^{3}$ mechanism). Funato et al. (2004) proposed a hybrid collision-capture mechanism. Initially two objects collide to produce a binary whose components (as is usual for a collision) have quite different masses. Subsequently, exchange "reactions" with larger third bodies displace the secondary and so ramp up the mass ratio. This eventually leads to binaries with similarly sized partners. However, this mechanism appears to lead to orbital properties (in particular, ellipticities) dissimilar to those actually observed (e.g., Noll 2003; Astakhov et al. 2005).

An additional capture scenario, chaos-assisted capture (CAC, see Astakhov et al. 2005; Lee et al. 2007), was originally proposed to explain the capture of irregular moons at the giant planets (e.g., Astakhov et al. 2003). The scenario for the formation of TNBs is as follows: two objects initially become caught up in very long-living, yet ultimately unstable, chaotic orbits within their mutual Hill sphere. During this phase, the binary may be permanently captured and subsequently hardened through multiple scattering encounters with relatively small "intruder" bodies. The CAC model predicts similarly sized binary components whose mutual orbits are in good agreement with observations. It has been indeed proposed that the propensity to form similarly sized binary partners is a direct fingerprint of chaos-assisted capture. A further specific prediction of the CAC mechanism concerns the distribution of retrograde and prograde mutual orbits, i.e., mutual orbit inclinations (Astakhov et al. 2005; Lee et al. 2007). In particular, retrograde mutual orbits are predicted to be relatively common. The CAC mechanism was criticized by Schlichting \& Sari (2008) who argue, e.g., that formation via transient, chaotic binaries is not as important as the $\mathrm{L}^{2}$ and $\mathrm{L}^{3}$ mechanisms. These objections are addressed in some detail by Gamboa Suárez et al. (2010).

Finally, Nesvorný et al. (2010) proposed a model in which TNBs formed during gravitational collapse. Angular momentum considerations in the planetesimal disk explain the formation of binaries rather than condensation into a single object. This gravitational instability model predicts identical compositions and colors for TNB partners and also inclinations of generally $i \leq 50^{\circ}$, i.e., retrograde mutual orbits are predicted to be rare. Unfortunately, true inclinations of mutual TNB orbits is currently available for a handful of systems only (see Grundy et al. 2011). For example, Table 5 and Figs. 5 and 6 show that only knowledge of the true inclination of the mutual orbit (i.e., that obtained by making further observations to remove the symmetry ambiguity) will help us determine whether the mutual orbit of the Orcus/Vanth system is either prograde or retrograde.

We note that the CAC model also allows for the direct formation of almost circular orbits similar to that of Orcus/Vanth. This mechanism was proposed as a possible origin of the apparently almost circular orbit of the TNB $2001 \mathrm{QW}_{322}$ (Petit et al. 2008; Gamboa Suárez et al. 2010). It is conceivable that the Orcus/Vanth system was produced in a similar manner. 
Table 6. Relative positions $(\delta x, \delta y)$ of Vanth with respect to Orcus for future occultation events ( $\delta x$ positive through East).

\begin{tabular}{lcc}
\hline \hline Date & $\delta x(\mathrm{~km})$ & $\delta y(\mathrm{~km})$ \\
\hline 2012 Mar 21 & -6977 & -5259 \\
2012 Nov 16 & -8937 & 347 \\
\hline
\end{tabular}

Notes. Uncertainty on position draws an ellipse of $\pm 450 \times 120 \mathrm{~km}$ on Earth, around the reported positions (see text for details).

In summary, determining the colors and compositions, as well as the true mutual orbital inclinations of TNBs (Schlichting \& Sari 2008) remain important quests. Knowledge of these properties will provide important information to constrain, and to distinguish between, the variously proposed TNB formation models.

\subsection{Stellar occultations}

Observations of stellar occultation phenomena are of great interest for deriving, in a direct and most precise way, the size of the occulting body. When several chords are gathered, one can derive the projected shape contour (see Millis \& Dunham 1989, for a extensive summary). Moreover, these observations help us to ascertain whether there is a teneous atmosphere, and then derive its pressure profile (e.g., Sicardy et al. 2003).

In the case of a binary system, it is also important to be able to predict the path of each component more than the one of the center of gravity (Assafin et al. 2010). Given the large separation of Vanth from Orcus $(\sim 9000 \mathrm{~km})$, the prediction of an event by the secondary can be either deplaced in time by several minutes when the binary system is oriented in the direction of apparent motion, or fully decoupled from the one of the primary otherwise. The occultation path of the primary might indeed be invisible from Earth while that of the secondary could be visible.

Owing to the current uncertainty in TNO ephemerides, a precise prediction of occultation requires "last-minute" astrometry measurements relative a to background star to obtain reliable predictions of the occultation track on Earth. In addition, the position of the secondary relative to the primary is needed to a precision of approximately $100 \mathrm{~km}$ to enable useful observational campaigns to be established. Given the improvement in the period estimate, and the orbital phase reset provided by our astrometric point obtained in 2010, we can derive more accurate prediction for the location of the secondary and subsequently for the track of stellar occultations by Vanth. Given the possible mass-ratio range, the path on the Earth of the primary can also be shifted from the position of the center-of-mass. Assuming that the components have the same albedo, the mass ratio is on the order of $\approx 0.02_{-0.02}^{+0.07}$, corresponding to a shift of $180_{-180}^{+630} \mathrm{~km}$. We list in Table 6 the relative position of Vanth around Orcus for upcoming stellar occultations. The relative positions in Table 6 corresponds to the nominal values; the associated error distribution is not exactly Gaussian, but an ellipse curved and stretched along the trajectory (similar to Fig. 5). The one $\sigma$ uncertainty is large, approximately $\pm 450 \mathrm{~km}$ along the secondary's nominal trajectory (about $\pm 120 \mathrm{~km}$ across), for both occultations. Depending on the occultation occurence (motion relative to the star and orientation of this uncertainty ellipse), it can correspond to a shift of the occultation path on Earth (or miss) or a shift in time. Updates are available online ${ }^{2}$.

\footnotetext{
${ }^{2}$ http://www.lesia.obspm.fr/perso/bruno-sicardy/
}

\section{Conclusion}

On the basis of spectro-imaging measurements of the OrcusVanth system, we have been able to obtain a spectrum of Orcus, measure the $H$ and $K$ magnitudes of Vanth, and determine their mutual relative positions. We have searched for possible weak bands in the spectrum of Orcus, limiting the possible amount of $\mathrm{CH}_{4}$ to no more than $\sim 2 \%$, and ethane to $\sim 5 \%$. The presence of other compound(s) is required to explain the depth of the absorption band detected at $2.2 \mu \mathrm{m}$. Presence of hydrated ammonia, or ammonium, could explain the observed band, but the lack of distinguishable features for these species in the observed wavelength range forbid any strong conclusion to be drawn.

Vanth has been found to be slightly redder than Orcus in the visible to near-infrared wavelength range, although uncertainties in the measurements are large. While this suggests that Vanth's composition, or age, is different from Orcus, it does not constrain formation scenarios (collision, co-formation, or capture). By detecting Vanth at another position three years after previous orbit calculations by Brown et al. (2010), we have been able to reset the phase of the orbit, allowing predictions of Vanth's track on Earth during future stellar occultation events.

Acknowledgements. We thank the staff of ESO's Paranal observatory for their assistance in obtaining this data. This research used the VO tool Miriade (Berthier et al. 2008) developed at IMCCE, and NASA's Astrophysics Data System. We acknowledge support from the Faculty of the European Space Astronomy Centre (ESAC) for F.D. visit. P.L. is grateful for the financial support of a Michael West Fellowship and of the Royal Society in the form of a Newton Fellowship. We thank our anonymous referee for their constructive comments.

\section{References}

Assafin, M., Camargo, J. I. B., Vieira Martins, R., et al. 2010, A\&A, 515, A32 Astakhov, S. A., Burbanks, A. D., Wiggins, S., \& Farrelly, D. 2003, Nature, 423, 264

Astakhov, S. A., Lee, E. A., \& Farrelly, D. 2005, MNRAS, 360, 401

Barucci, M. A., Brown, M. E., Emery, J. P., \& Merlin, F. 2008a, The Solar System Beyond Neptune, 143

Barucci, M. A., Merlin, F., Guilbert, A., et al. 2008b, A\&A, 479, L13

Berthier, J., \& Marchis, F. 2001, BAAS, 33, 1049

Berthier, J., Hestroffer, D., Carry, B., et al. 2008, LPI Contributions, 1405, 8374

Bonnet, H., Abuter, R., Baker, A., et al. 2004, The Messenger, 117, 17

Britt, D. T., Yeomans, D. K., Housen, K. R., \& Consolmagno, G. J. 2002, Asteroids III, 485

Brown, M. E., van Dam, M. A., Bouchez, A. H., et al. 2006, ApJ, 639, 43

Brown, M. E., Ragozzine, D., Stansberry, J., \& Fraser, W. C. 2010, AJ, 139, 2700 Carry, B., Vernazza, P., Dumas, C., \& Fulchignoni, M. 2010, Icarus, 205, 473

Cook, J. C., Desch, S. J., Roush, T. L., Trujillo, C. A., \& Geballe, T. R. 2007, ApJ, 663, 1406

Cooper, J. F., Christian, E. R., Richardson, J. D., \& Wang, C. 2003, Earth Moon and Planets, 92, 261

Cottin, H., Moore, M. H., \& Bénilan, Y. 2003, ApJ, 590, 874

de Bergh, C., Delsanti, A., Tozzi, G. P., et al. 2005, A\&A, 437, 1115

Delsanti, A., Merlin, F., Guilbert-Lepoutre, A., et al. 2010, A\&A, 520, A40

DeMeo, F. E., Fornasier, S., Barucci, M. A., et al. 2009, A\&A, 493, 283

DeMeo, F. E., Barucci, M. A., Merlin, F., et al. 2010, A\&A, 521, A35

Dumas, C., Carry, B., Hestroffer, D., \& Merlin, F. 2011, A\&A, 528, A105

Eisenhauer, F., Abuter, R., Bickert, K., et al. 2003, SPIE, 4841, 1548

Ferraz-Mello, S., Rodríguez, A., \& Hussmann, H. 2008, Celest. Mech. Dyn. Astron., 101, 171

Fornasier, S., Dotto, E., Barucci, M. A., \& Barbieri, C. 2004, A\&A, 422, L43

Funato, Y., Makino, J., Hut, P., Kokubo, E., \& Kinoshita, D. 2004, Nature, 427, 518

Gamboa Suárez, A., Hestroffer, D., \& Farrelly, D. 2010, Celest. Mech. Dyn. Astron., 106, 245

Goldreich, P., Lithwick, Y., \& Sari, R. 2002, Nature, 420, 643

Grundy, W. M., \& Schmitt, B. 1998, J. Geophys. Res., 103, 25809

Grundy, W. M., Noll, K. S., Nimmo, F., et al. 2011, Icarus, 213, 678

Guilbert, A., Alvarez-Candal, A., Merlin, F., et al. 2009, Icarus, 201, 272 
B. Carry et al.: Integral-field spectroscopy of (90482) Orcus-Vanth

Hapke, B. 1993, Theory of reflectance and emittance spectroscopy (Cambridge University Press)

Hestroffer, D., Vachier, F., \& Balat, B. 2005, Earth Moon and Planets, 97, 245

Hilton, J. L. 2002, Asteroids III, 103

Khare, B. N., Sagan, C., Arakawa, E. T., et al. 1984, Icarus, 60, 127

Khare, B. N., Thompson, W. R., Cheng, L., et al. 1993, Icarus, 103, 290

Kozai, Y. 1962, AJ, 67, 591

Lee, E. A., Astakhov, S. A., \& Farrelly, D. 2007, MNRAS, 379, 229

Levi, A., \& Podolak, M. 2009, Icarus, 202, 681

Lim, T. L., Stansberry, J., Müller, T. G., et al. 2010, A\&A, 518, L148

Markwardt, C. B. 2009, in ASP Conf. Ser. 411, ed. D. A. Bohlender, D. Durand, \& P. Dowler, 251

Merline, W. J., Weidenschilling, S. J., Durda, D. D., et al. 2002, Asteroids III, 289

Millis, R. L., \& Dunham, D. W. 1989, Asteroids II, 148

Modigliani, A., Hummel, W., Abuter, R., et al. 2007

[arXiv:astro-ph/0701297]

Müller, T. G., Lellouch, E., Böhnhardt, H., et al. 2009, Earth Moon and Planets, 105, 209

Murray-Clay, R. A., \& Schlichting, H. E. 2011, ApJ, 730, 132
Nesvorný, D., Youdin, A. N., \& Richardson, D. C. 2010, AJ, 140, 785

Noll, K. S. 2003, Earth Moon and Planets, 92, 395

Ortiz, J. L., Cikota, A., Cikota, S., et al. 2011, A\&A, 525, A31

Petit, J.-M., Kavelaars, J. J., Gladman, B. J., et al. 2008, Science, 322, 432

Quirico, E., \& Schmitt, B. 1997, Icarus, 127, 354

Schaller, E. L., \& Brown, M. E. 2007, ApJ, 659, L61

Schlichting, H. E., \& Sari, R. 2008, ApJ, 673, 1218

Schmitt, B., Quirico, E., Trotta, F., \& Grundy, W. M. 1998, in Solar System Ices,

ed. B. Schmitt, C. de Bergh, \& M. Festou, Astrophys. Space Sci. Libr., 227, 199

Sicardy, B., Widemann, T., Lellouch, E., et al. 2003, Nature, 424, 168

Stansberry, J., Grundy, W., Brown, M. E., et al. 2008, The Solar System Beyond Neptune, 161

Strazzulla, G., \& Palumbo, M. E. 1998, Planet. Space Sci., 46, 1339

Thirouin, A., Ortiz, J. L., Duffard, R., et al. 2010, A\&A, 522, A93

Trujillo, C. A., Brown, M. E., Rabinowitz, D. L., \& Geballe, T. R. 2005, ApJ, 627,1057

Tryka, K. A., Brown, R. H., Chruikshank, D. P., et al. 1994, Icarus, 112, 513

Weidenschilling, S. J. 2002, Icarus, 160, 212 